\title{
Mobilização terapêutica como \\ cuidado de enfermagem: evidência surgida da prática*
}

\author{
THERAPEUTIC MOBILIZATION AS NURSING CARE: EVIDENCE FROM PRACTICE \\ MOVILIZACIÓN TERAPÉUTICA COMO CUIDADO DE ENFERMERÍA: \\ EVIDENCIA SURGIDA DE LA PRÁCTICA
}

\author{
Renata Flavia Abreu da Silva1', Maria Aparecida de Luca Nascimento²
}

\section{RESUMO}

Estudo observacional e descritivo que teve como objetivo identificar como os parâmetros relativos à mecânica pulmonar do paciente crítico, sob ventilação mecânica, se comportam, após o procedimento técnico de mudança de decúbito realizado pela equipe de enfermagem. A população acessível, inserida por amostragem não-probabilística, foram 9 pacientes críticos, sob ventilação mecânica, e seus respectivos parâmetros referentes à mecânica pulmonar. Foram observadas alterações positivas e negativas na mecânica pulmonar em todos os pacientes, em diferentes posições, possivelmente associada à mudança de decúbito. Conclui-se que a avaliação clínica é fundamental para a prescrição de enfermagem relativa à mudança de decúbito, pois a mecânica pulmonar pode se modificar de acordo com a posição do paciente no leito, trazendo, inclusive, resultados negativos.

\section{DESCRITORES}

Cuidados de enfermagem

Respiração artificial

Mecânica respiratória

Posicionamento do paciente

Modalidades de posição

\begin{abstract}
The objective of this observational and descriptive study was to identify the behavior of relative parameters related to pulmonary mechanics of critical patients undergoing mechanic ventilation, after the nursing staff performed the technical procedure of decubitus position change. The accessible population, by non-probabilistic sampling, consisted of nine critical patients undergoing mechanical ventilation and their respective parameters related to pulmonary mechanics. Positive and negative alterations in pulmonary mechanics in all patients were identified, correlating with different positions, possibly associated with the change in decubitus position. In conclusion, clinical assessment is essential in nursing care regarding decubitus position change, because pulmonary mechanics may change according to the patient's position in bed, and may result in negative consequences.
\end{abstract}

\section{DESCRIPTORS \\ Nursing care \\ Respiration, artificial \\ Respiratory mechanics \\ Patient positioning \\ Modalities, position}

\section{RESUMEN}

Estudio observacional y descriptivo que tuvo como objetivo: identificar el modo en que los parámetros relativos a la mecánica pulmonar del paciente crítico, bajo ventilación mecánica, se comportan luego del procedimiento técnico de cambio de decúbito realizado por el equipo de enfermería. La población con acceso, inserta por muestra no probabilística, fue de nueve pacientes críticos, bajo ventilación mecánica, y sus respectivos parámetros referentes a la mecánica pulmonar. Fueron observadas alteraciones positivas y negativas en la mecánica pulmonar en todos los pacientes, en diferentes posiciones, posiblemente asociada al cambio de decúbito. Se concluyó en que la evaluación clínica es fundamental para la prescripción de enfermería relativa al cambio de decúbito, pues la mecánica pulmonar puede modificarse de acuerdo con la posición del paciente en el lecho, trayendo, inclusive, resultados negativos

\section{DESCRIPTORES}

Atención de enfermería Respiración artificial Mecánica respiratoria Posicionamiento del paciente Modalidades de posición

\footnotetext{
* Extraído da dissertação "Mobilização terapêutica como cuidado da enfermagem: evidência surgida na prática", Centro de Ciências Biológicas e da Saúde Universidade Federal do Estado do Rio de Janeiro, 2008. ${ }^{1}$ Mestre em Enfermagem. Enfermeira Gerente de Risco do Instituto Nacional de Cardiologia Brasileiro. Rio de Janeiro, RJ, Brasil. rflavia@gmail.com. ${ }^{2}$ Enfermeira. Doutora em Enfermagem. Orientadora Acadêmica do Programa de Pós-Graduação em Enfermagem da Universidade Federal do Estado do Rio de Janeiro. Rio de Janeiro, RJ, Brasil. gemeas@centroin.br
} 


\section{INTRODUÇÃO}

As Unidades de Terapia Intensiva (UTI) abrigam pacientes que, momentaneamente, necessitam de uma assistência mais complexa, devido ao seu estado clínico de natureza mais crítica, cuidados altamente complexos e controles estritos ${ }^{(1)}$. Verifica-se então que a enfermagem envolve-se neste processo prestando uma assistência com características específicas, pois o conhecimento adquirido na UTI se destaca, sobretudo, pelo avanço progressivo e rápido dos novos conhecimentos na área, bem como pelo aparato tecnológico presente nestas unidades ${ }^{(1)}$.

As UTI constituem um conjunto de elementos funcionalmente agrupados que exigem além de equipamentos, assistência médica e de enfermagem ininterruptas e especializadas. Sendo assim, o cuidado, baseado no julgamento e conhecimento clínico, implementado pelo enfermeiro objetiva estabelecer intervenções que possam abranger as necessidades bio-psico- sociais dos pacientes $^{(1)}$

Algumas intervenções da equipe de enfermagem possuem objetivos específicos, como por exemplo, a mudança de decúbito, que normalmente é referenciada como uma forma de prevenção das úlceras por pressão(2).

Uma das causas das úlceras por pressão é a imobilidade do paciente no leito sem a possibilidade de movimentar-se por si próprio, dependendo de mobilização passiva $^{(2-5)}$.

Além da imobilidade dos pacientes no leito, outros fatores também contribuem para o surgimento das úlceras por pressão, como por exemplo, o uso de fármacos vasoativos, vasculopatia, extremos de peso, hiper e hipotermia, alterações circulatórias, incontinência, déficit imunológico, umidade, extremos de idade, alterações do turgor ${ }^{(3-4)}$.

Com objetivo de prevenir a problemática supracitada, passou-se ao uso de estratégias sistematizadas como a estratificação dos fatores de risco, proteção da pele contra as forças de cisalhamento e, por fim, a mudança do decúbito, que tem por objetivo diminuir a pressão em pontos mais suscetíveis de maneira rotineira e padroni$z^{2} a^{(2)}$. Entretanto, mesmo com variadas causas contribuintes para o surgimento de uma úlcera por pressão, enfatiza-se que a mudança de decúbito, função precípua da equipe de enfermagem, não poderá, sob hipótese alguma, ser negligenciada ${ }^{(5)}$.

Dentre as estratégias adotadas para sistematizar o posicionamento do paciente no leito, destaca- se um método, comumente utilizado, baseado no funcionamento de um relógio. Neste método, a cada duas horas, o decúbito é modificado em dorsal, lateral direito e esquerdo para aliviar a pressão nos tecidos. Esta técnica difundiu-se amplamente, passando a ser utilizada em inúmeros centros de tratamento para pacientes acamados e sem mobilidade ativa(2).

Ao associar o procedimento técnico em foco à finalidade para a qual, normalmente, ele é direcionado, verifica-se que seus benefícios não se restringem apenas à prevenção das úlceras por pressão, mas também, à prevenção da síndrome de desuso muscular ${ }^{(6-8)}$, ao conforto ${ }^{(9)}$, e à interferência no padrão respiratório com a otimização da relação ventilação/perfusão(10-14) do paciente.

O paciente acamado pode apresentar várias complicações, dentre elas, a síndrome do desuso muscular ${ }^{(7)}$, sendo a mudança de decúbito uma das intervenções a serem realizadas, objetivando-se a prevenção desta síndrome e sua repercussão sistêmica(6-7). Dentre as repercussões sistêmicas destacam-se as úlceras por pressão, constipação, estase de secreções pulmonares, trombose, infecção do trato urinário, retenção urinária, força ou resistência diminuída, hipotensão ortostática, desorientação, distúrbio da imagem corporal e sentimento de impotência ${ }^{(6-7)}$. O desuso muscular pode contribuir ainda para o prolongamento do tempo de internação do paciente, aumentando, inclusive, sua exposição a possíveis fatores agravantes ${ }^{(6-7)}$. Cabe salientar que na realidade brasileira, as intervenções sistemáticas realizadas para a prevenção da síndrome do desuso muscular são atribuições, comumente, realizadas pelo fisoterapeuta. Contudo, os autores deste estudo consideram que, os profissionais da equipe de enfermagem, ao promoverem a mudança de decúbito dos pacientes sob os seus cuidados, interferem em sua posição no leito, contribuindo, consequentemente, para a prevenção da referida síndrome ${ }^{(6)}$.

Considerando que o presente estudo tem como foco o paciente em estado crítico, e internado em UTI, observa-se que, comumente, ele está sendo ventilado mecanicamente. Deste modo, a sua mobilização possui extrema importância, pois auxilia na drenagem das secreções das vias aéreas inferiores, facilitando a aspiração traqueal ${ }^{(8,15)}$. Além disso, a mobilização adequada auxilia no processo do desmame da ventilação mecânica, proporcionando a melhora da relação ventilação/perfusão (V/Q) do tecido pulmonar, diminuindo a resistência ao trabalho muscular, e contribuindo assim para reduzir a sensação de dispnéia( ${ }^{(8,14-15)}$. O decúbito pode influenciar na mecânica pulmonar, aumentando o trabalho respiratório e modificando a relação ventilação/perfusão dependendo da posição no leito e do grau de elevação do dorso $^{(9-14)}$. O conhecimento acerca da mudança de decú- 
bito e seus efeitos na oxigenação do paciente constituem saber essencial para enfermeiras que cuidam de pacientes em situação crítica $^{(10)}$.

Apreende-se então que a mudança de decúbito, sendo relevante cuidado de enfermagem, envolve várias ciências, demonstrando que por mais simples que possa parecer, possui determinada complexidade ${ }^{(5)}$.

No cotidiano da prática do cuidado em UTI pode-se observar assistematicamente que algumas posições, durante a mudança de decúbito no paciente em ventilação mecânica, provocam a alteração do seu padrão respiratório, conforme constatado através de mudança dos parâmetros no ventilador mecânico. Buscando na fisiologia a explicação para esta situação verifica-se que dependendo do quadro clínico do paciente, o decúbito interfere diretamente no aumento do consumo de oxigênio e maior trabalho respiratório(9-12,16-17).

A monitorização da mecânica pulmonar se faz necessária durante a assistência de enfermagem, pois o enfermeiro ao cuidar de um paciente em ventilação mecânica precisa estar sob vigilância constante ${ }^{(8,18)}$. Isso inclui a monitorização contínua, que atualmente é propiciada pela alta tecnologia, presente nas máquinas e equipamentos disponíveis no mercado. Por isso, o enfermeiro necessita associar conhecimentos de mecânica e de clínica respiratória aos seus conhecimentos de fisiologia ${ }^{(8,18)}$. Enfatiza-se que as propriedades da mecânica do sistema respiratório podem ser facilmente monitorizadas à beira de leito. Parâmetros como volume corrente, fluxo e pressão do sistema respiratório, resistência das vias aéreas e complacência pulmonar, podem ser obtidos através de um monitor gráfico e um sensor de fluxo acoplado às vias aéreas artificiais ou do pneumotacógrafo(18).

Aplicando os conceitos e estudos, anteriormente citados, à prática cotidiana da equipe de enfermagem, tem-se como pressuposto que o procedimento técnico de mudança de decúbito do paciente crítico sob ventilação mecânica influencia diretamente na sua mecânica pulmonar, necessitando, portanto, do julgamento clínico do enfermeiro para poder ser realizado.

Desse modo, considerando que a mudança de decúbito é uma tecnologia inserida na prática assistencial da equipe de enfermagem, com a finalidade de prevenir as úlceras por pressão, pretendeu-se com o presente estudo alcançar o seguinte objetivo:

Identificar como os parâmetros relativos à mecânica pulmonar do paciente crítico, sob ventilação mecânica, se comportam, após o procedimento técnico de mudança de decúbito realizado pela equipe de enfermagem.

Desta forma, o presente estudo pretende trazer elementos a serem considerados pelo enfermeiro que, baseando o seu cuidado em um julgamento clínico, poderá, inclusive, determinar se a mudança de decúbito do paciente crítico sob ventilação mecânica em uma UTI se faz necessária, considerando que certos procedimentos podem, se mal aplicados, passarem, de um fator coadjuvante, a um fator complicador do tratamento.

\section{MÉTODO}

Estudo observacional e descritivo ocorrido em uma unidade cardio-intensiva clínica de hospital da esfera federal, de referência em cardiologia, no município do Rio de Janeiro.

Os critérios de inclusão foram: pacientes com idade acima de 18 anos, em ventilação mecânica, no modo ventilatório assisto-controlado, Escala de Sedação de Ramsay entre 5 e 6 , onda de fluxo decrescente e Volume Corrente (VC) programado entre 4 e $8 \mathrm{~mL} / \mathrm{Kg}$. O único critério de exclusão foi a não aceitação do familiar em autorizar a mensuração dos parâmetros dos pacientes.

O referido estudo atendeu às especificações da resolução 196/96 e ao Código de Ética de Enfermagem, sendo submetido ao Comitê de Ética e Pesquisa da instituição em apreço, através do qual foi aprovado em 22 de outubro de 2007 sob o número 0167/12.09.07.

A amostra do estudo constituiu de 09 pacientes críticos, em ventilação mecânica e seus parâmetros referentes à mecânica pulmonar, sendo mostrados neste estudo cinco gráficos gerados da coleta de dados, associados aos parâmetros de 04 pacientes. Os pacientes foram inseridos no estudo por amostragem não-probabilística por conveniência com população acessível.

\section{Coleta e apresentação dos dados}

Os dados foram coletados nos momentos em que o paciente era reposicionado nas mudanças de decúbito: lateral direito, lateral esquerdo e dorsal. As mensurações ocorreram antes e após a mudança do decúbito, nos minutos, 2, 15, 60 e 120 da realização e o apareIho de ventilação utilizado foi o microprocessado BIRD8400ST.

As variáveis coletadas foram: IMC, sexo, tipo de prótese (tubo orotraqueal ou traqueostomia), diagnóstico radiológico do tórax e as referentes diretamente à mecânica pulmonar como Volume Corrente, Pressão Expiratória Final Positiva (PEEP), Pressão de Pico, Pressão de Platô, além de Saturação Periférica de Oxigênio $\left(\mathrm{SpO}_{2}\right)$, e os sinais vitais Frequência Respiratória (FR), Frequência Cardíaca (FC) e Pressão Arterial (PA).

Dos dados coletados, foram utilizados três parâmetros para avaliação dos pacientes: VC, Complacência Estática (Cst) calculada através da Pressão de Platô e PEEP e $\mathrm{SpO}_{2}$, por refletir melhor as mudanças que ocorrem no parênquima pulmonar ${ }^{(17)}$. 
Os pacientes foram nomeados por letras A, B, C e D e a apresentação dos dados foi realizada por meio de estatística descritiva exemplificada em gráficos.

\section{RESULTADOS}

Os pacientes que compuseram a amostra do estudo apresentavam cardiopatia descompensada e eram, em sua maioria, homens (67\%), apresentavam sobrepeso (67\%) e eram idosos (89\%).

Cabe salientar que os parâmetros respiratórios avaliados não sofreram interferência de otimização da oxigenação, pois durante todo o período de coleta de dados, nem a Fração Inspirada de Oxigênio $\left(\mathrm{FiO}_{2}\right)$ e nem a Pressão Expiratória Final Positiva (PEEP) foram modificadas.

A paciente A (Figura 1) era idosa, apresentava sobrepeso, estava intubada e há doze dias em ventilação mecânica, e não apresentava alteração em radiografia de tórax. No momento da coleta de dados, a referida paciente A estava em decúbito lateral esquerdo e foi posicionada em decúbito lateral direito. A FR manteve-se estável, assim como FC e PA, porém podemos observar na Figura 1 que o VC, a Cst e a $\mathrm{SpO}_{2}$ melhoraram após a mudança de decúbito e, se mantiveram estáveis até duas horas após.

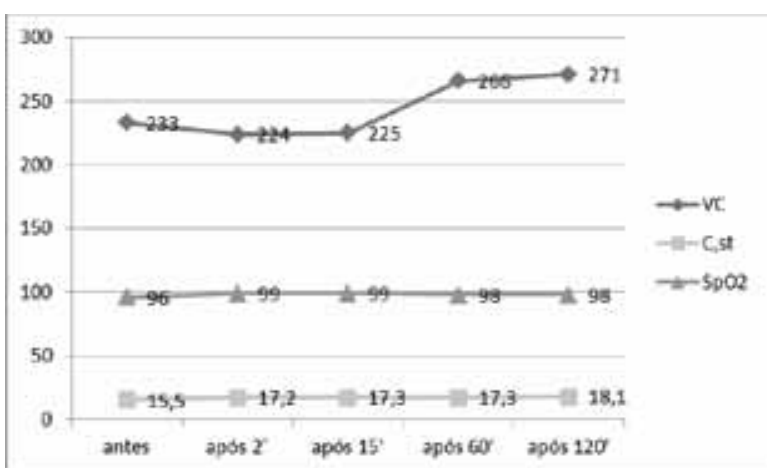

Figura 1 - Distribuição dos parâmetros referentes à Mecânica Pulmonar da paciente A - Rio de Janeiro, 2008

A paciente $B$ (Figura 2) também era idosa e apresentava sobrepeso, estava traqueostomizada e há nove dias em ventilação mecânica, sem alteração em radiografia de tórax. No momento da coleta de dados, ela estava em decúbito lateral esquerdo e foi posicionada em decúbito dorsal elevado em 30 graus aproximadamente. As FR e FC mantiveram-se estáveis e houve melhora na PA com diminuição da pressão arterial sistólica. A figura 2 mostra o VC, a Cst e a $\mathrm{SpO}_{2}$ e o principal fato relevante a ser observado é que após duas horas estes parâmetros não só melhoraram, como mantiveram-se estáveis.

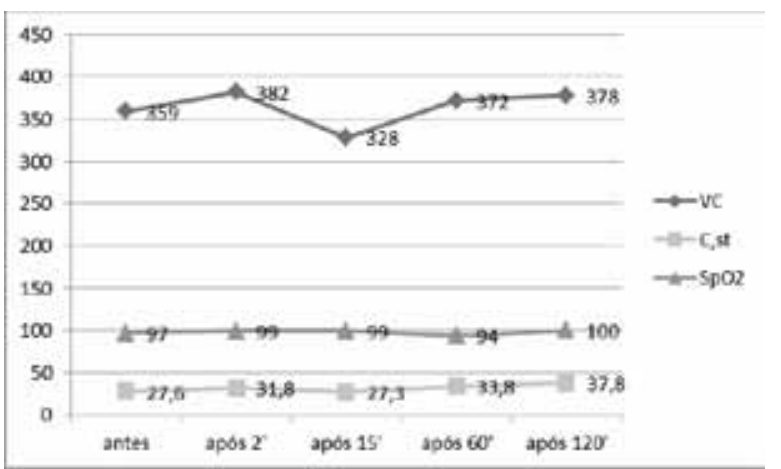

Figura 2 - Distribuição dos parâmetros referentes à Mecânica Pulmonar da Paciente B - Rio de Janeiro, 2008

As Figuras 3 e 4 representam o paciente $C$ que teve, inicialmente, seu decúbito modificado de lateral esquerdo para decúbito lateral direito. $O$ paciente $C$ apresentava sobrepeso, era idoso, estava traqueostomizado e estava há dez dias em ventilação mecânica, FC e FR estavam em parâmetros normais e no momento da coleta de dados não apresentava alterações em radiografia de tórax. Observa-se que o VC aumentou após a mudança de decúbito, refletindo na Cst, e, consequentemente, na $\mathrm{SpO}_{2}$.

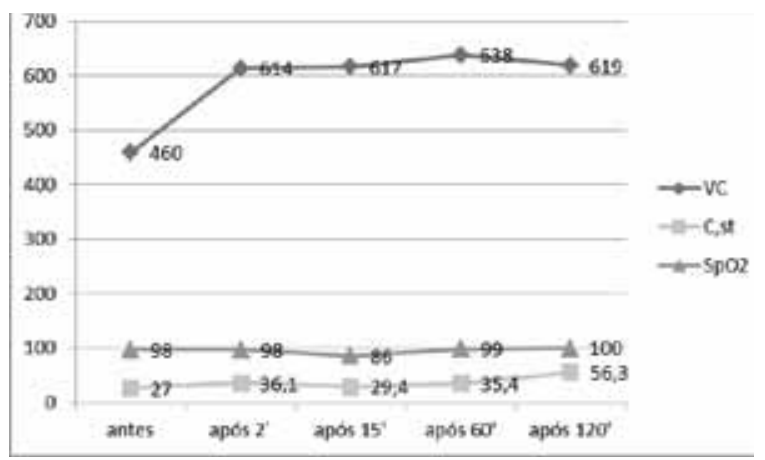

Figura 3 - Distribuição dos Parâmetros Referentes à Mecânica Pulmonar do Paciente C - Rio de Janeiro, 2008

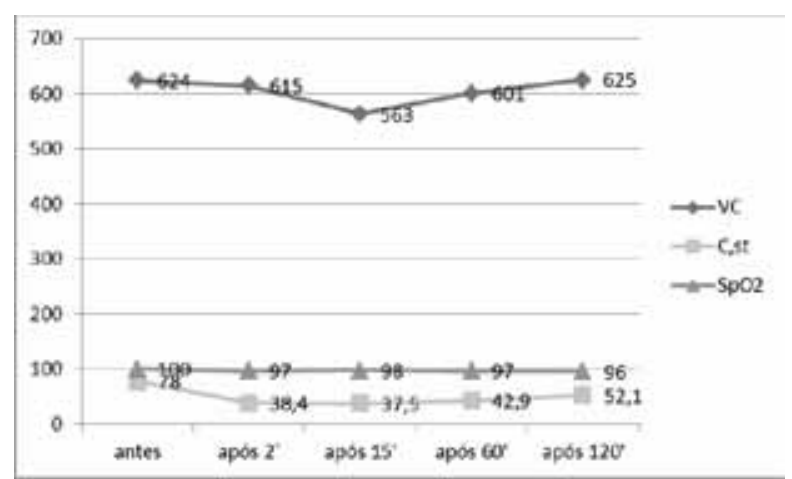

Figura 4 - Distribuição dos Parâmetros Referentes à Mecânica Pulmonar do Paciente C - Rio de Janeiro, 2008 
O paciente $\mathrm{C}$ foi posicionado de decúbito lateral direito para decúbito lateral esquerdo e após ser mobilizado manteve o VC, porém apresentou queda na Cst e na $\mathrm{SpO}_{2}$.

A paciente $D$ era idosa, obesa e apresentou recidivas de derrames pleurais, líquidos entre as pleuras visceral e parietal, o que levou à pleurodese como conduta por parte do pneumologista que estava acompanhando o caso. Não havia no prontuário descrição do tipo de pleurodese realizada, apenas o pulmão afetado: o direito.

No momento da avaliação a referida paciente estava em decúbito lateral direito e foi posicionada sobre decúbito dorsal elevado a aproximadamente 30 graus. Observa-se na figura 5 o grande incremento no VC, assim como a $\mathrm{SpO}_{2}$ e a Cst. Vale salientar que a paciente em questão apresentava taquipnéia antes da mudança de decúbito, $\mathrm{e}$ que houve melhora deste parâmetro após a mobilização.

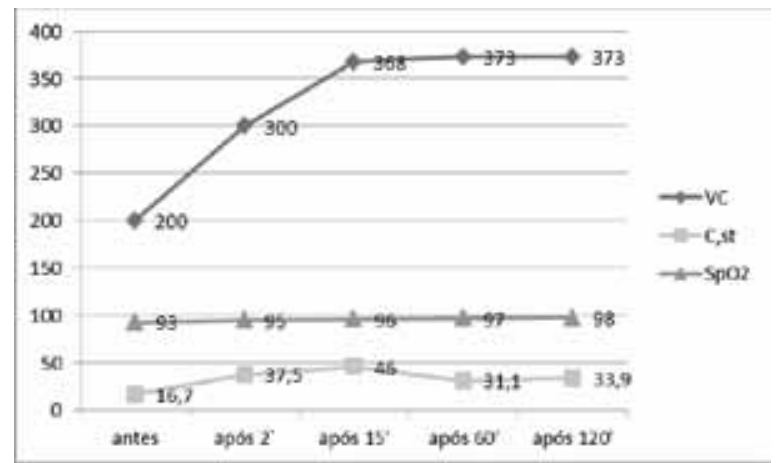

Figura 5 - Distribuição dos Parâmetros Referentes à Mecânica Pulmonar da Paciente D - Rio de Janeiro, 2008.

\section{DISCUSSÃO}

Os dados iniciais conferem a estes pacientes uma pré-disposição a evoluir com lesões como úlceras por pressão e, portanto, necessitam de mudanças de decúbito sistemáticas ${ }^{(19-20)}$. Todavia, estes mesmos pacientes apresentam características contra indicativas do posicionamento sobre decúbito dorsal no leito em supina, como idade, doença cardiopulmonar e obesidade ${ }^{(17)}$, evidenciando a avaliação clínica necessária antes da prescrição da mudança de decúbito.

A oferta de oxigênio ao organismo depende da inter-relação entre sistemas respiratório e cardiovascular ${ }^{(17)}$, todavia a avaliação hemodinâmica sistemática não foi realizada durante coleta de dados neste estudo.

Por mais que a ventilação mecânica objetive a melhora do paciente, ela poderá causar eventos adversos, pelo fato de não ser fisiológica, e isto deve ser levado em consideração ao se modificar a posição do paciente acamado, considerando que as zonas pulmonares serão afetadas. Além disso, a posição corporal influi na distribuição da ventilação, da perfusão, tamanho dos alvéolos, mecânica respiratória e oxigenação arteria|(10,16,21-22).
Em determinado estudo ${ }^{(21)}$, após avaliação da hemodinâmica e da oxigenação dos pacientes em função do posicionamento sobre decúbitos laterais, foi identificada como primordial a avaliação individual do paciente e sua resposta à mobilização.

A Figura 1 mostra que ser posicionada sobre o decúbito lateral direito foi melhor para a paciente $A$, porque ele manteve-se hemodinamicamente estável e com os seus parâmetros ventilatórios inalterados. Isso pode ser explicado pela propriedade do pulmão direito, por ser, anatomicamente, maior que o esquerdo ${ }^{(11-12)}$, e consequentemente mais perfundido. Por isso, a lateralização, se escolhida, deve ser baseada nesta premissa, dentre as outras avaliações necessárias.

Outro aspecto a se considerar é o protocolo de mudança de decúbito que preconiza sua realização a cada duas horas ${ }^{(2,7,15)}$. No entanto, conforme mostram as Figuras 1 e 2, no momento em que a mecânica pulmonar das pacientes $A$ e B, respectivamente, alcançou uma estabilidade, elas serão novamente mobilizadas de acordo com a rotina. Será que esta mudança de decúbito a ser realizada seria benéfica para elas sob o aspecto respiratório? Por que mobilizá-la se o padrão respiratório está estável?

Conforme já descrito, a mobilização do paciente no leito influencia o aspecto respiratório ${ }^{(11-14)}$, podendo ou não ser benéfica a ele. Contudo, considerando que a técnica em apreço é realizada no cotidiano da prática apenas para prevenção de úlceras por pressão ${ }^{(2,4)}$, observa-se que esta influência não está sendo cogitada. Isso demonstra a necessidade de se utilizar o raciocínio clínico como guia para priorizar os cuidados aos pacientes. Isto é, considerando que mobilizar o paciente no leito possui outros benefícios, além da prevenção de úlceras por pressão, há de se considerar e priorizar esses benefícios, antes da prescrição da mudança de decúbito.

Além disso, existem outras estratégias para prevenção de úlceras por pressão, além da mudança de decúbito ${ }^{(2,5)}$, e elas podem e devem ser consideradas, principalmente quando mobilizar envolve instabilidade respiratória e hemodinâmica ${ }^{(16,21)}$ e, consequentemente, perda da estabilidade clínica e possível dano ao paciente.

Outro aspecto a ser levado em consideração é a afirmativa de que a propriedade anatômica proporciona vantagem ao pulmão direito na mobilização lateral poderia ser utilizada para explicar o ocorrido com o paciente $C_{\text {, }}$ Figuras 3 e 4, ao ser posicionado sobre decúbitos laterais diferentes ${ }^{(11-12)}$.

Observa-se ainda a necessidade de constatar-se a presença de doença uni ou bilateral nos pulmões do paciente a ser mobilizado, isto é, a posição lateral somente será terapêutica se observadas alterações pulmonares antes da mobilização ${ }^{(23)}$. Isso foi evidenciado no caso da paciente $D$ que estava posicionada sobre um decúbito que, conforme sua história clínica seria contraindicado para ela, e isto foi 
evidenciado após a mudança de decúbito, que, nesta situação, foi terapêutica, mesmo não intencional.

Em doença unilateral, na maioria dos casos, prevalece o adágio, pulmão sadio para baixo ${ }^{(10-12)}$; contudo, alguns autores questionam tal argumentação, salientando que a avaliação individual do paciente é o que determinará a posição a ser adotada ${ }^{(13-14,21,23)}$, fato este, corroborado pelo presente estudo. Observou-se em determinado estudo ${ }^{(16)}$ que em pacientes em pós-operatório de cirurgia torácica, quando o pulmão operado era colocado para baixo, ocorria a piora nas trocas gasosas, e quando o pulmão sadio estava voltado para baixo, havia aumento na $\mathrm{PaO}_{2}$, porém não significativa.

O posicionamento do paciente em decúbito lateral é uma forma de terapia não-invasiva, todavia, pode contribuir para a deterioração do status respiratório do paciente, se a posição for aplicada indiscriminada ou inapropriadamente ${ }^{(10,16,21)}$.

\section{Algumas considerações em relação às posições:}

Dorsal (Supina) $\rightarrow$ Diminui diâmetro ântero-posterior no tórax e proporciona aumento no diâmetro lateral, ocorrendo, por sua vez o deslocamento cranial do diafragma devido a pressão intra-abdominal aumentada. Além disso, aumenta o volume sanguíneo torácico, levando à diminuição da Capacidade Residual Funcional (CRF), diminuição da complacência pulmonar e aumento do trabalho respiratório e maior demanda de oxigênio; contraindicada, principalmente, em tabagistas, obesos e idosos ${ }^{(17)}$.

Ventral (Prona) $\rightarrow$ Determina efeitos favoráveis, pois o diafragma apresenta melhor excursão na posição prona devido sua porção posterior ter um raio menor de curvatura, gerando maior pressão transdiafragmática e maior alongamento do músculo(10,17) .

Lateral $\rightarrow$ Pode ser benéfico ou deletério, dependendo da avaliação individual do paciente; no decúbito lateral ocorre maior excursão na hemicúpula diafragmática dependente, pelo alongamento destas fibras com o deslocamento cefálico e com isso, o deslocamento antero-posterior do abdome diminui a expansão da caixa torácica pela diminuição da base de apoio do diafragma para realizar este movimento ${ }^{(17)}$.

Com o objetivo de verificar se a mobilização será terapêutica, critérios deverão ser adotados cada vez que o paciente for posicionado de forma diferente. Os parâmetros do ventilador mecânico que devem ser verificados, em associação com a monitorização disponível podem ser: oximetria, capnografia, ventilometria, pressão arterial, eletrocardiograma e gasometria arterial ${ }^{(13)}$.

O posicionamento corporal a ser adotado para o paciente deve ser eleito conforme os objetivos fisiológicos e terapêuticos a fim de aliviar a dispnéia, evitar a trepopnéia, aumentar a oxigenação e melhorar a ventilação. Tais considerações podem ser confirmadas pela seguinte afirmação ${ }^{(13)}$ : Não existe posição ideal, a resposta clínica do paciente é soberana.

\section{CONCLUSÃO}

Após a mobilização no leito, foram observadas alterações na mecânica pulmonar de todos os pacientes a ela submetidos. Algumas foram evidentes, outras sutis, e, mesmo com um número pequeno de pacientes, foi possível evidenciar aspectos fundamentais a serem considerados antes de realizar o referido procedimento técnico.

Ao cuidar de pacientes críticos em ventilação mecâni$\mathrm{ca}$, o enfermeiro encontra-se diante do desafio de manter a qualidade da assistência prestada, e evitar os danos advindos deste cuidado, os chamados eventos adversos.

Considerando que o cuidado prioriza, primordialmente, a manutenção da vida com qualidade, a mobilização realizada de forma terapêutica interfere em um dos mecanismos mais intrínsecos do indivíduo, a respiração, que é um dos parâmetros que constitui a avaliação da presença de vida. Seria ousadia nossa, inferir que a mobilização, ao ser praticada terapeuticamente, intervém na manutenção da vida, uma vez que a mecânica pulmonar otimizada auxilia na saída do paciente da ventilação mecânica e pode até postergar o suporte ventilatório invasivo?

Diante dos resultados obtidos, pode-se afirmar que ao influenciar a mecânica pulmonar de pacientes críticos em ventilação mecânica, a mudança de decúbito, realizada de forma crítica e por meio de julgamento clínico, torna-se uma mobilização terapêutica.

O procedimento técnico de mudança de decúbito, realizado pela equipe de enfermagem sob prescrição ou indicação do enfermeiro, envolve mais fatores do que simplesmente mudar a posição do paciente no leito. Sua influência na mecânica pulmonar, conforme mostrado no presente estudo evidencia que esta técnica não deve ser realizada de forma rotineira. Por isso, ao mobilizar o paciente no leito objetivando a prevenção das úlceras por pressão, os outros aspectos advindos desta mobilização devem ser considerados.

O presente estudo não esgota, e nem tampouco, responde a todas as questões referentes à influência da mobilização do paciente na mecânica pulmonar, mas já evidencia que esta mobilização pode ser ou não terapêutica. Demonstra ainda que mais estudos acerca deste tema são necessários, de forma a visualizar o cuidado de enfermagem sob o ponto de vista clínico, criando evidências que possam baseá-lo num cuidado crítico, prescrito individualmente, conforme a clínica do paciente, mesmo na vigência de protocolos.

Conclui-se que ao agir dessa forma, evitando os eventos adversos da mobilização do paciente critico, deixaremos de fazer, simplesmente, uma mudança de decúbito e realizaremos, com consciência e cientificidade, uma mobilização terapêutica. 


\section{REFERÊNCIAS}

1. Viana RAPP, Whitaker IY. Enfermagem em terapia intensiva: práticas e vivências. Porto Alegre: Artmed; 2011.

2. Dealey C. Cuidando de feridas. 3a ed. São Paulo: Atheneu; 2008.

3. Rogenski NMB, Santos VLCG. Estudo sobre a incidência de úlceras por pressão em um Hospital Universitário. Rev Latino Am Enferm [Internet]. 2005 [citado 2008 maio 30];13(4). Disponível em: http://www.scielo.br/pdf/rlae/v13n4/v13n4a03. pdf

4. Maklebust J. Pressure ulcers: the great insult. Nurs Clin North Am. 2005;40(2):365-89.

5. Nascimento MAL, Blank M, Barros MCD, Guedes MTS. Colchão de segmentos: uma tecnologia para o cuidado de enfermagem nas úlceras por pressão. In: Anais do 12 Pesquisando em Enfermagem; 2005 maio 9-12; Rio de Janeiro [CD-ROM]. Rio de Janeiro: Escola de Enfermagem Anna Nery; 2005.

6. McCloskey JC, Bulechek GM. Classificação das intervenções de enfermagem. 4a ed. Porto Alegre: Artmed; 2008.

7. Carpenito-Moyet LJ. Diagnóstico de enfermagem: aplicação à prática clínica. 13a ed. São Paulo: Artmed; 2006.

8. Ashurst S. Cuidados de enfermagem de doentes ventilados mecanicamente em UCl: 1 e 2. Nursing (São Paulo). 1998;3(120):20-7.

9. Moore T. The Effect of lateral positioning on oxygenation in acute unilateral lung disease. Nurs Crit Care. 2002;7(2): 278-82.

10. Marklew A. Body positioning and its effect on oxygenation: a literature review. Nurs Crit Care. 2006;11(1):16-22.

11. Rowat A. Patient positioning and its effect on brain oxygenation. Nurs Times. 2001; 97(43):30-2.

12. Lasater-Erhard M. The effect of patient position on arterial oxygen saturation. Crit Care Nurse. 1995;15(5):31-6.

13. Azeredo C. A. Ventilação mecânica invasiva e não-invasiva. Rio de Janeiro: Revinter; 1994.
14. Yeaw EMJ. The effect of body positioning upon maximal oxygenation of patients with unilateral lung pathology. J Adv Nurs. 1996;23(1):55-61.

15. Mozachi N. Cuidados gerais. In: Souza VHS, Mozachi N. O hospital: manual do ambiente hospitalar. 2a ed. Curitiba: Manuel Real; 2006. p. 46-78.

16. Pimenta CAM. Efeitos das posições corporais sobre os gases do sangue arterial em portadores de disfunção pulmonar: estudo em pacientes em pós-operatório de cirurgia cardíaca [dissertação]. São Paulo: Escola de Enfermagem, Universidade de São Paulo, 1988.

17. Carvalho CRR. II Consenso Brasileiro de Ventilação Mecânica. Rio de Janeiro: Atheneu; 2000. p. 387-9.

18. Silva RFA, Barreiro Filho RD, Nascimento MAL. Monitorizando a mecânica respiratória durante assistência de enfermagem: buscando evidências na literatura. Enferm Atual. 2010;59(5):18-20.

19. Ursi ES, Galvão CM. Prevenção de lesões de pele no perioperatório: revisão integrativa da literatura. Rev Latino Am Enferm. 2006;14(1):124-31.

20. Gomes FSL, Bastos MAR, Matozinhos FP, Tempone HR, Velásquez-Meléndez $\mathrm{G}$. Factors associated to pressure ulcers in patients at Adult Intensive Care Units. Rev Esc Enferm USP [Internet]. 2010 [cited 2011 Jan 23];44(4):1070-6. Available from: http://www.scielo.br/pdf/reeusp/v44n4/en_31.pdf

21. Banasik JL, Emerson RJ. Effect of lateral positions on tissue oxygenation in the critical ill. Heart Lung. 2001;30(4): 269-76.

22. Hewitt N, Bucknall T. The respiratory and haemodynamic response to lateral patient positioning in critically ill patients [Internet]. [cited 2008 Oct 14]. Available from: http://www. joannabriggs.edu.au/protocols/resphaemo.pdf

23. Kim MJ, Hwang $\mathrm{HJ}$, Song $\mathrm{HH}$. A randomized trial on the effects of body positions on lung function with acute respiratory failure patients. Int J Nurs Stud. 2002;39(5):549-55. 